\title{
TENERIFE TIENE SEGURO DE SOL (Y DE LUNA): REPRESENTACIONES DEL PROFESORADO DE PRIMARIA ACERCA DEL DÍA Y LA NOCHE
}

\author{
VEGA NAVARRO, ANA ${ }^{1}$ \\ Centro Superior de Educación. Universidad de La Laguna \\ E-mail: amvega@ull.es
}

\begin{abstract}
SUMMARY
This study is adressed to report which the ideas about «day» and «night» are from a sample of Primary School Teachers in the Island of Tenerife. A frequent association of the Moon with the night is persistently shown by a third part of teachers. As a consequence, they consider the Moon to be static, and therefore are not able to correctly explain the day and night cycle. Four wrong representations are identified: rotation, revolution, appearance-disappearance and occultation-eclipse.

In the light of these results, the author points out the need for revising textbooks contents and images and giving a new direction to teacher's education. Thus, scientific and didactics education has to take into account that it is actually inside the classrooms where pupils learn many of their alternative frameworks transmitted by their own teachers.
\end{abstract}

\section{INTRODUCCIÓN}

El desarrollo de las representaciones infantiles relativas al origen de los astros y del universo, así como las causas y la naturaleza de la noche comenzó a ser estudiado en la década de los veinte por Piaget (1926), pero fue escaso el interés mostrado por otros investigadores hasta época reciente. Los estudios de Klein (1982), Sadler (1987), Jones, Lynch y Reesink (1987), Baxter (1989), Vosniadou y Brewer (1990, 1994), Finegold y Pundak (1991), Schoon (1992), Osborne y otros (1994), Afonso y otros (1995), Resines (1995), Sharp (1996), Samarapungavan, Vosniadou y Brewer (1996), Diakidoy, Vosniadou y Hawks (1997), Fleer (1997), entre otros, han venido a poner de manifiesto que son muchas las maneras que los niños y las niñas, de diferentes edades y culturas, tienen para explicar el día y la noche: unas veces el Sol desaparece cuando llegan para taparlo las nubes, la noche o la Luna; otras es el Sol quien se desplaza para situarse detrás de las nubes y de la Luna o para esconderse en un lugar indeterminado del cielo, detrás de las montañas o en el mar. Con frecuencia se hace girar al Sol cada día en torno a la Tierra, moviéndose hacia arriba y hacia abajo, y aun acercándose y alejándose y que la Luna lo siga; o que la Tierra se desplace diariamente alrededor del Sol, permaneciendo estáticos la Luna y el Sol. No es raro que el alumnado conozca la existencia de un movimiento de rotación terrestre, pero tampoco lo es que entienda que la Luna y el Sol se encuentran estáticos en posiciones diametralmente opuestas, para explicar que el día es un producto del Sol y la noche de la Luna.

Las ideas del profesorado en activo han sido menos estudiadas que las del alumnado. La investigación de Jones (1988) daba cuenta de las ideas que algunos estudiantes de magisterio de Tasmania tenían en relación con el ciclo día-noche, las fases lunares y la estaciones, igual que hicieron Dai y Capie (1990), en Taiwan, y Mant y Summers (1993), con estudiantes de magisterio americanos, y más recientemente Parker y Heywood 
(1998) y Dickinson, Flick y Lederman (1998). Camino (1991) hizo un estudio comparativo de esos tres fenómenos con estudiantes de magisterio argentinos, que posteriormente le sirvió para detectar las ideas previas de un grupo de maestros en ejercicio y diseñar una unidad didáctica con la que intentar cambiarlas (Camino, 1995). Por su parte, después de presentar las ideas de algunos futuros maestros de Kentucky en relación con el ciclo día-noche y las estaciones, Atwood y Atwood (1995, 1997) mostraron que un breve tiempo de instrucción con maestros de enseñanza elemental puede servir para mejorar la comprensión de esos fenómenos. El cambio conceptual de estudiantes de magisterio en relación con estos conceptos es objeto de estudio de la asignatura Didáctica de las Ciencias de la Facultad de Educación de Cádiz (Navarrete, 1998).

En este trabajo se da cuenta de las ideas acerca del día y la noche sostenidas por un grupo de maestros de primaria en ejercicio de la isla de Tenerife, intentando contribuir a ampliar la literatura científica y didáctica, bastante escasa en España. Se pretende, por otra parte, llamar la atención sobre la necesidad de una urgente reorientación de la didáctica de las ciencias en relación con la formación del profesorado. Una formación que no puede estar solamente centrada en cuestiones metodológicas y en el tópico constructivista del cambio conceptual del alumnado, olvidando que, como ya planteó Schoon (1995), muchas de las ideas alternativas del alumnado se originan en las aulas porque esas ideas son las que tiene el profesorado.

\section{MUESTRA Y METODOLOGÍA}

El profesorado participante fue reclutado en tres cursos de formación que no estaban directamente relacionados con los aspectos aquí tratados, pues versaban sobre cuestiones organizativas y metodológicas de la educación infantil. Por esa razón solamente nos centramos en conocer y describir las ideas del profesorado sin plantear intervención alguna. En total colaboraron 96 profesores (87 mujeres y 9 varones), casi todos interinos con destino en Tenerife en diferentes cursos de primaria y con varios años de docencia (entre 2 y 12 años; media 5,6).

Se pasó un cuestionario que los profesores contestaron de forma individual con lápiz y papel durante un tiempo aproximado de 90 minutos. Como puede verse en el anexo, el cuestionario constaba de cuatro tareas, cuyos ítems fueron tomados de investigaciones anteriores y utilizados de forma parecida (Nussbaum y Novak, 1976; Baxter, 1989; Vosniadou y Brewer, 1994; Sharp, 1996; Sneider y Ohadi, 1998).

\section{RESULTADOS}

En los dibujos realizados en la primera tarea, la Tierra aparece generalmente redonda, lo cual no siempre significa que se conciba esférica. Como puede advertirse en
Tabla I

Forma de la Tierra, de acuerdo con el dibujo de la tarea 1

\begin{tabular}{|lc|}
\hline a) Redonda & 93 \\
\hline b) Plana & 2 \\
\hline c) Semiesférica & 1 \\
\hline
\end{tabular}

la tabla I hay dos profesores que la dibujan plana: son los profesores núm. 4 y 65, que, como veremos (Fig. 11 y 14), tienen una representación no científica del día y la noche. Un profesor la representa redonda con una línea que la divide por el medio: cree, probablemente, que la Tierra es la semiesfera que está debajo de la línea y que la parte que está por encima es la cúpula celeste, como parece confirmar posteriormente en la tarea 4.1 , ya que es uno los dos que figura en la alternativa $b$ de la tabla II.

Tabla II

Forma de la Tierra, teniendo en cuenta la tarea 4.1

\begin{tabular}{|lc|}
\hline $\begin{array}{l}\text { a) Esférica, pero nos parece plana porque sólo vemos } \\
\text { una pequeña parte }\end{array}$ & 82 \\
\hline $\begin{array}{l}\text { b) Esférica, pero vivimos en el centro en una zona plana } \\
\text { c) Dos Tierras diferentes: la esférica del espacio } \\
\text { y plana donde vivimos }\end{array}$ & 2 \\
\hline $\begin{array}{l}\text { d) Esférica con partes planas, que es donde vivimos } \\
\text { e) Redonda alrededor y plana por encima, } \\
\text { en donde vivimos }\end{array}$ & 3 \\
\hline f) No contesta & 6 \\
\hline
\end{tabular}

En la tabla II aparece la forma que los profesores atribuyeron a la Tierra de acuerdo con la pregunta 1 de la tarea 4. La alternativa $b$, Tierra esférica pero vivimos en el centro en una zona plana, manifestada por dos profesores, viene a decirnos que la Tierra es una semiesfera recubierta por la bóveda celeste. Uno de esos dos profesores es el que la dibujaba semiesférica en la tarea 1 de la tabla I. La alternativa $c$, dos Tierras diferentes: la del espacio y la plana en donde vivimos es la que sostiene el profesor núm. 70, que tiene una representación que hemos llamado de ocultamiento y eclipse (Fig. 15). El profesor 4, que la dibujaba plana y mantiene una representación de aparición y desaparición del Sol y la Luna (Fig. 14), está incluido en la alternativa $d$ : esférica con partes planas, que es donde vivimos. Otro que aparece en esta categoría es el núm. 96, que la dibujó redonda en la tarea 1 pero mantiene una representación de revolución, semejante a la del profesor 65. Cinco profesores de la categoría e, redonda alrededor y plana por encima, dibujaron una Tierra redonda en la tarea 1 pero sus explicaciones son escasas o incoherentes (están incluidos en lo que hemos denominado representación inco- 
herente, igual que uno de la categoría $f$, porque en realidad piensan que la Tierra es redonda y plana como una galleta).

Tabla III

Situación de la Luna, de acuerdo con el dibujo y la asociación a la noche.

\begin{tabular}{|l|l|}
\hline $\begin{array}{l}\text { Asociada a la explicación de la noche y dibujada } \\
\text { frente al Sol }\end{array}$ & 31 \\
\hline $\begin{array}{l}\text { Asociada a la explicación de la noche y dibujada } \\
\text { pero no frente al Sol. }\end{array}$ & 6 \\
\hline $\begin{array}{l}\text { Asociada a la explicación de la noche, } \\
\text { pero no se dibuja }\end{array}$ & 7 \\
\hline $\begin{array}{l}\text { No está asociada a la explicación de la noche, } \\
\text { pero aparece dibujada. }\end{array}$ & 7 \\
\hline $\begin{array}{l}\text { No está asociada a la explicación de la noche y no } \\
\text { aparece dibujada }\end{array}$ & 39 \\
\hline \begin{tabular}{l} 
Dibujos dudosos o incoherentes \\
\hline
\end{tabular}
\end{tabular}

La tabla III nos indica qué vinculación tiene la Luna con la noche, de acuerdo con el dibujo realizado y con la asociación que han hecho en sus explicaciones. Debe tenerse en cuenta que esa asociación no siempre es equivalente a una explicación no científica del ciclo díanoche. Es decir, que hay profesores que explican el día y la noche como la ausencia y presencia de la luz solar derivada de la rotación terrestre, aunque después dibujan la Luna. Y al contrario, los hay que no dibujan la Luna, pero que explican que el día y la noche tienen que ver con la presencia alternativa del Sol y de la Luna.

Tabla IV

¿Qué es lo que verás sin duda alguna en el cielo en una noche de verano?

\begin{tabular}{|lc|}
\hline Estrellas (cielo estrellado, a veces nubes o planetas) & 50 \\
\hline La Luna y las estrellas & 32 \\
\hline La Luna & 4 \\
\hline Otros (oscuridad, contaminación, cielo, etc. ) & 10 \\
\hline
\end{tabular}

En la tabla IV aparecen las respuestas a la primera pregunta de la tarea 2: ¿Qué verás en el cielo en una noche de verano? En bastantes casos responden de forma instintiva que verán la Luna y las estrellas. Pero muchos lo hacen de forma reflexiva: cuando están seguros de ver la Luna en cualquier momento de la noche es porque para ellos la presencia de la Luna es condición de la noche. Son casi todos aquéllos que en la tabla 10 afirman que la Luna no se mueve y que en la tabla 8 sostienen que por el día está en el otro lado de la Tierra, en donde es de noche, escondida en el horizonte o no contestan.
Tabla V

¿Dónde está el Sol (en esa noche de verano)?

\begin{tabular}{|lc|}
\hline Al otro lado, lado opuesto (de nosotros, de la Tierra) & 35 \\
\hline En el otro hemisferio & 3 \\
\hline En otra parte, en donde es de día & 7 \\
\hline En su sitio (el de siempre, el mismo que por el día) & 6 \\
\hline Oculto & 16 \\
\hline No lo vemos (no está, desapareció) & 11 \\
\hline $\begin{array}{l}\text { Se escondió (se fue) (en el oeste 2; este 1; norte 1; } \\
\text { izquierda 1; a dormir 1) }\end{array}$ & 6 \\
\hline En el espacio, en el cielo & 7 \\
\hline Detrás de la Luna & 2 \\
\hline Girando alrededor de la Tierra & 1 \\
\hline No contesta & 2 \\
\hline
\end{tabular}

La tabla V nos dice dónde está el Sol por la noche, de acuerdo con las respuestas de la tarea 2 , pregunta $b$. De una u otra manera, la mayoría afirma que está en la otra parte de la Tierra, en donde es de día, debido a la rotación terrestre. Dos profesores lo sitúan detrás de la Luna porque para ellos la noche se produce precisamente cuando el Sol queda tapado por ella. Hay otro que señala que desapareció, aunque considera también que está detrás de la luna.

Tabla VI

¿Cómo ocurre eso?

\begin{tabular}{|lc|}
\hline $\begin{array}{l}\text { Por la rotación (giro, movimiento sobre sí misma) } \\
\text { de la Tierra }\end{array}$ & 60 \\
\hline La Tierra gira (se traslada) alrededor del Sol & 9 \\
\hline La rotación y la traslación & 3 \\
\hline $\begin{array}{l}\text { Por los movimientos (posiciones) del Sol, la } \\
\text { Luna y la Tierra }\end{array}$ & 4 \\
\hline Porque el Sol se oculta por un lugar y sale por el otro & 2 \\
\hline Porque ha venido la noche & 2 \\
\hline Porque estamos (está la Tierra) de espaldas & 3 \\
\hline Porque la Tierra tapa con su cuerpo los rayos solares & 1 \\
\hline Porque el Sol ilumina otra parte de la Tierra & 2 \\
\hline Respuestas evasivas o no contestan & 10 \\
\hline
\end{tabular}

Las tablas VI y VII nos aportan información acerca de las razones que se atribuyen a la desaparición del Sol durante la noche, de acuerdo con las respuestas a la tarea 2 , pregunta $c$. Solamente hay 60 que manifiestan ex- 
presamente que ello se deriva de la rotación de la Tierra, pero lo cierto es que son más de 80 los que tienen esa idea. En ese sentido, sólo dos de los 9 que afirman que el Sol desaparece porque la Tierra gira a su alrededor tienen realmente esa explicación, los otros 7 identifican correctamente que el ciclo día-noche se deriva de la rotación, aunque usen inapropiadamente el lenguaje o incorporen la traslación a la explicación del ciclo día-noche.

Tabla VII

¿Qué debe ocurrir para que se haga de día dentro de unas horas?

\begin{tabular}{|lc|}
\hline Que la Tierra gire (siga girando, rotando) & 62 \\
\hline Que la Tierra se ponga de cara al Sol & 3 \\
\hline Que el Sol salga & 6 \\
\hline Que el Sol y la Tierra realicen sus movimientos & 2 \\
\hline $\begin{array}{l}\text { Que se vaya aproximando el Sol (donde estoy, } \\
\text { donde vivo, enfrente) }\end{array}$ & 5 \\
\hline Que pasen las horas, esperar a que amanezca & 5 \\
\hline Que haya movimiento & 1 \\
\hline Que la Luna se vaya y aparezca el Sol & 1 \\
\hline Que se oculte la Luna detrás de nubes & 1 \\
\hline Que la Luna se esconda detrás del Sol & 1 \\
\hline Que la Tierra gire alrededor del Sol & 2 \\
\hline No contesta & 7 \\
\hline
\end{tabular}

La mayoría entiende que el paso de la noche al día es una consecuencia de la rotación de la Tierra, si bien a veces se explica de diferentes maneras, y a veces en términos vagos o inapropiados, derivados del uso de expresiones cotidianas, como se muestra en la tabla VII. Así, de entre los 6 que manifiestan que es necesario que el Sol salga y de los 5 que afirman que el Sol debe aproximarse a nosotros, solamente hay dos que realmente lo atribuyen al movimiento real del Sol. Los dos que en esta tabla VII mantienen que de día la Luna se esconde (detrás de las nubes o del Sol) son los que afirmaban en la tabla $\mathrm{V}$ que durante la noche el Sol está detrás de la Luna, lo cual indica que ésa es la representación mental que tienen.

En la tabla VIII se recogen las respuestas dadas a la pregunta 4 de la cuarta tarea. Las respuestas «al otro lado», «en otro sitio», «en donde es de noche», «oculta», «durmiendo», nos señalan la estrecha vinculación que para muchos profesores tienen la Luna y la noche.

Las tablas IX, X y XI recogen las respuestas dadas a la pregunta $e$ de la tarea 2. La mayor parte de los profesores afirman que el Sol no se mueve, y es de suponer, aunque no podemos asegurarlo en todos los casos, que quieren decir que no es un movimiento real del Sol lo que explicaría el ciclo día-noche. En ese sentido, probable-
Tabla VIII

¿Dónde está la Luna durante el día?

\begin{tabular}{|lc|}
\hline Al otro lado (del Sol, de la Tierra) & 13 \\
\hline En donde es de noche & 15 \\
\hline Oculta & 11 \\
\hline En otro sitio & 7 \\
\hline No está & 5 \\
\hline Depende (de la hora, de su movimiento, de la órbita) & 34 \\
\hline Está durmiendo (3) o escondida en el horizonte (3) & 6 \\
\hline No contesta & 5 \\
\hline
\end{tabular}

Tabla IX

¿El Sol se mueve?

\begin{tabular}{|lc|}
\hline No & 67 \\
\hline Sí & 12 \\
\hline Sí, pero es otro movimiento & 6 \\
\hline Creo que no & 2 \\
\hline Creo que sí & 1 \\
\hline No contesta & 5 \\
\hline
\end{tabular}

Tabla X

¿La Luna se mueve?

\begin{tabular}{|lc|}
\hline No & 32 \\
\hline Sí & 42 \\
\hline Sí, con la Tierra & 5 \\
\hline Sí, alrededor de la Tierra & 9 \\
\hline No contesta & 8 \\
\hline
\end{tabular}

Tabla XI

¿La Tierra se mueve?

\begin{tabular}{|lc|}
\hline Sí & 93 \\
\hline No & 2 \\
\hline No contesta & 1 \\
\hline
\end{tabular}


mente bastantes de ellos conocen la rotación solar y su traslación alrededor del centro de la galaxia, como ocurre con los 6 que afirman que se trata de «otro movimiento». Es posible, incluso, que algunos de los que afirman escuetamente que sí se mueve se estén refiriendo precisamente a ello. Queremos destacar, y ésa era la razón de la pregunta, que aquellos profesores que tienen lo que llamamos representación de revolución o de aparición y desaparición afirman que el Sol sí se mueve y quieren señalar con ello que ese movimiento explica en parte el día y la noche.

Es posible que el alto número de profesores que afirma que la Luna no se mueve esté afirmando eso en relación con su explicación del día y la noche, aun cuando fuera de ese contexto pudieran decir lo contrario. De la misma forma, algunos de los que afirman que sí se mueve no están haciendo referencia a su movimiento mensual, sino a un supuesto movimiento diario que viene a explicar la llegada de la noche. De todas maneras, los conflictos en relación con el movimiento o no de la Luna son habituales, igual que ocurre con otras investigaciones, que ponen de manifiesto que sólo una pequeña parte del profesorado es capaz de explicar las fases lunares (12\%, Cohen, 1982; 3\%, Jones, 1988; 10\%, Mant y Summers, 1993).

\section{DISCUSIÓN}

\section{¿Modelos mentales o representaciones?}

Como hemos podido advertir, son muchas las incoherencias e incorrecciones manifestadas por bastantes profesores a la hora de explicar el ciclo día-noche. En primer lugar, existe una forma inapropiada de referirse al movimiento de la Tierra que explique el día y la noche: casi una decena afirma que se debe a su translación alrededor del Sol. También se suele atribuir un movimiento erróneo al Sol: que salga, que se vaya acercando, etc. Sin embargo, esas inapropiadas expresiones no siempre condicionan la explicación correcta del ciclo día-noche

Sí resultan decisivas las creencias expuestas en las tablas III, IV, VIII y X de que la Luna está inmóvil en el lado de la Tierra en donde no es de día y que puede verse en cualquier momento de la noche. Eso es lo que impide a casi una treintena de profesores explicar correctamente el ciclo. Y es de suponer, aunque no lo hemos analizado, que todos ellos tendrán dificultades para explicar las fases lunares y la naturaleza de eclipses y mareas.

Decisiva resulta también la dificultad que tiene más de una decena de profesores para hacer compatible la Tierra esférica con la Tierra aparentemente plana que todos los días pisan, como se muestra en la tabla II, pues prácticamente ninguno, en su fuero interno, asocia la rotación de la Tierra con el día y la noche, por más que hayan oído y estudiado que la Tierra rota.

Las concepciones e ideas del profesorado de nuestra muestra no difieren sustancialmente de las encontradas en otros estudios, como los de Jones (1988), Mant y
Summers (1993), Camino (1991, 1995), Schoon (1995) y Atwood y Atwood (1995). Y concuerdan, por otra parte, con las ideas infantiles de las investigaciones reseñadas en la introducción y con los modelos mentales propuestos por Vosniadou y Brewer (1994).

El modelo mental, tal y como lo conciben estos autores, sería una estructura condicionada por creencias, inferencias, presuposiciones y otras estructuras mentales, formada en las mentes infantiles en el intento de reconciliar, de manera lenta y gradual pero coherente, las creencias y presuposiciones experienciales con los datos e informaciones recibidas durante la escolaridad y del contacto con el mundo adulto. Se trata, por tanto, de estructuras mentales dinámicas que evolucionan a medida que nuevas informaciones se confrontan con las creencias y presuposiciones que conforman los modelos mentales previos y menos desarrollados.

Nosotros llamamos representaciones y no modelos mentales al conjunto de ideas del profesorado porque creemos que no son construcciones dinámicas ni evolutivas: cuando son incoherentes provienen de un bloqueo conceptual que el profesorado arrastra desde su época infantil. Mientras que el modelo mental que un niño posee le sirve para organizar coherentemente sus ideas en relación con sus creencias y principios epistemológicos, sin cuestionar si es alternativo o si concuerda con el modelo científico, las representaciones incoherentes del profesorado bloquean el ámbito de conocimiento del que forman parte, porque el profesorado sí es consciente de esa incoherencia.

De acuerdo con los dibujos y las respuestas, creemos haber identificado en el profesorado las cinco representaciones explicativas del fenómeno día-noche que se exponen en la tabla XII. Esas representaciones, basadas en la forma de entender la Tierra, en las explicaciones para situar a la Luna y al Sol por el día y por la noche y en los movimientos otorgados tanto a la Tierra como al Sol y a la Luna, están ilustradas con dibujos de los propios profesores.

Tabla XII

\begin{tabular}{|lr|}
\hline Representación del ciclo día-noche & Núm. de profesores \\
\hline A. Representación científica & 53 \\
\hline B. Representación rotacional & 28 \\
\hline C. Representación de revolución & 5 \\
\hline D. Representación de aparición y desaparición & 1 \\
\hline E. Representación de ocultamiento y eclipse & 3 \\
\hline F. Incoherentes o insuficiente explicación & 6 \\
\hline
\end{tabular}

\section{A. Representación científica}

Para estos 53 profesores, el día y la noche tienen que ver con la ausencia-presencia de la luz solar y eso se deriva 


\section{INVESTIGACIÓN DIDÁCTICA}

de la rotación de la Tierra, que hace que una parte esté iluminada por el Sol y la otra no, como dibuja y explica el profesor 89. Para todos ellos, la Tierra es esférica, aunque aparece plana porque sólo vemos una pequeña parte. Casi la mitad (26) de esos 53 profesores no hacen ninguna referencia a la Luna para explicar el ciclo díanoche y tampoco la hacen aparecer en sus dibujos. Todos ellos entienden que la Luna se mueve, y muchos explican que lo hace alrededor de la Tierra cada 28 días.

No es frecuente que las estrellas se distribuyan por todo el espacio, como hacen estos profesores: generalmente aparecen en la parte de la noche. Es raro (jsólo hay 3!) que tengan un elaborado concepto de la gravedad en relación con la dirección de la lluvia hacia el centro de la Tierra, como se muestra en el dibujo del profesor 23. Eso contrasta con los hallazgos de Sharp (1996), de quien hemos tomado la tarea, puesto que en su trabajo parece ser que la mitad de los niños de 10 y 11 años realizan dibujos semejantes o equivalentes. Eso no quiere decir, no obstante, que sean únicamente 3 los profesores que pueden dar cuenta correctamente de la gravedad terrestre. Solamente significa que existe una gran dificultad para realizar un dibujo en el que los objetos y los cuerpos estén orientados hacia el centro de la Tierra, como le ocurre, en parte, al profesor núm. 23: tiene una explica- ción correcta de la gravedad, del ciclo día-noche y de las estaciones; no es mal dibujante y orienta correctamente el agua cayendo, pero no es capaz de hacer lo mismo con las personas.

Aparecen tres variantes de la representación científica, en función de que la Luna se asocie o no a la noche, aun cuando la explicación del ciclo día-noche sea correcta.

\section{Variante a (13 de los 53)}

La Luna no interviene ni en los dibujos ni en las explicaciones, pero aseguran que la verán sin duda mirando al cielo en una noche estrellada. En este caso, la respuesta relativa a la Luna aparece de forma más instintiva que reflexiva.

\section{Variante b (9 de los 53)}

En sus explicaciones escritas, que son correctas, los profesores incluidos en esta variante no hacen referencia a la Luna. Si miran al cielo en una noche cualquiera, están seguros de ver estrellas, pero no mencionan la Luna. Sin embargo, la Luna aparece frente al Sol en sus dibujos. Es verdad que durante el día -y durante la noche- nuestro satélite puede estar en cualquier lugar de

Figuras 1 y 2

Modelo científico explicativo de los profesores 89 y 23, respectivamente. Si hubieran dibujado la Luna, como hacen algunos otros, podrían situarla en cualquier parte de su órbita mensual.
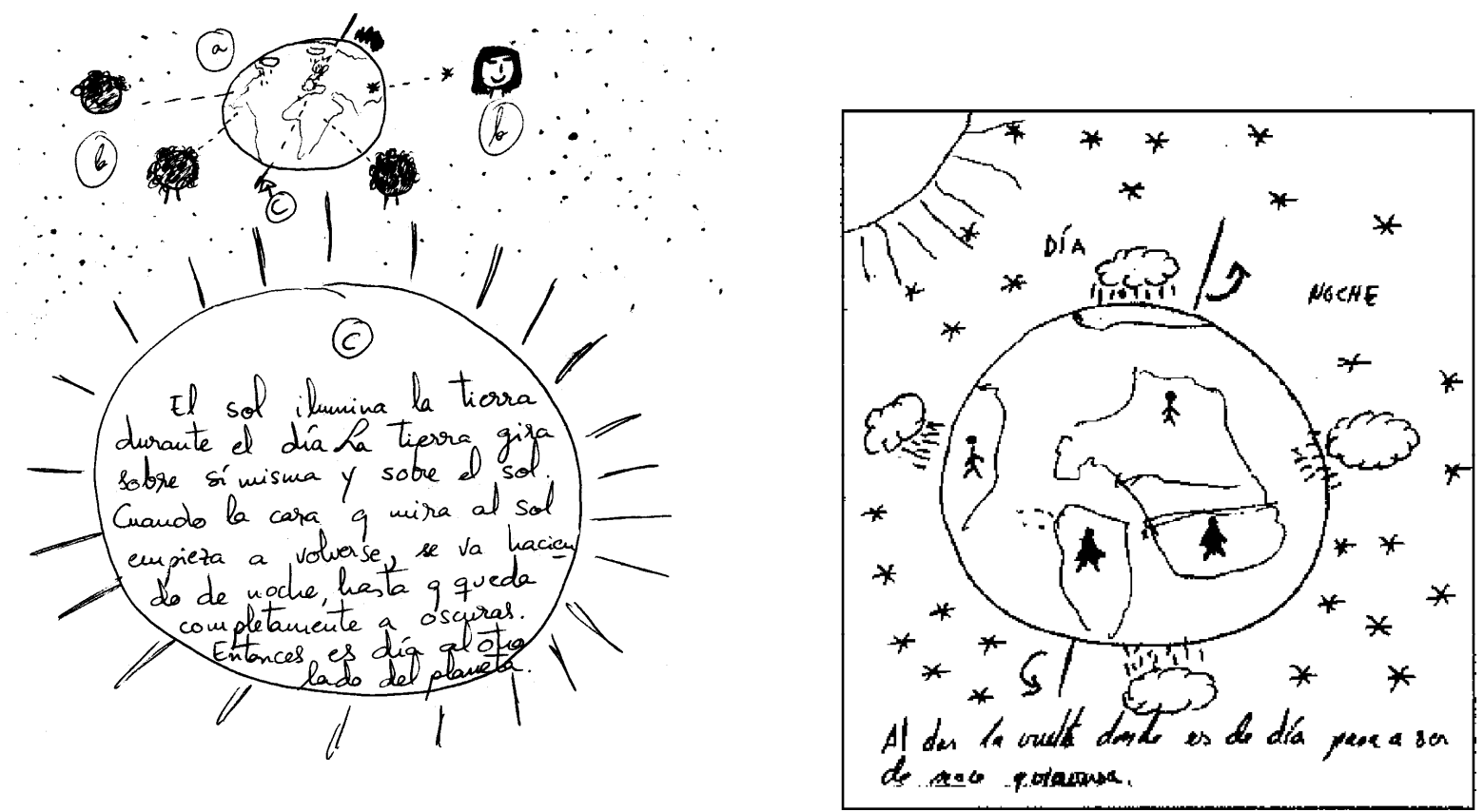
Figuras 3 y 4

Modelo científico, variante $a$, de los profesores 6 y 84 , respectivamente.
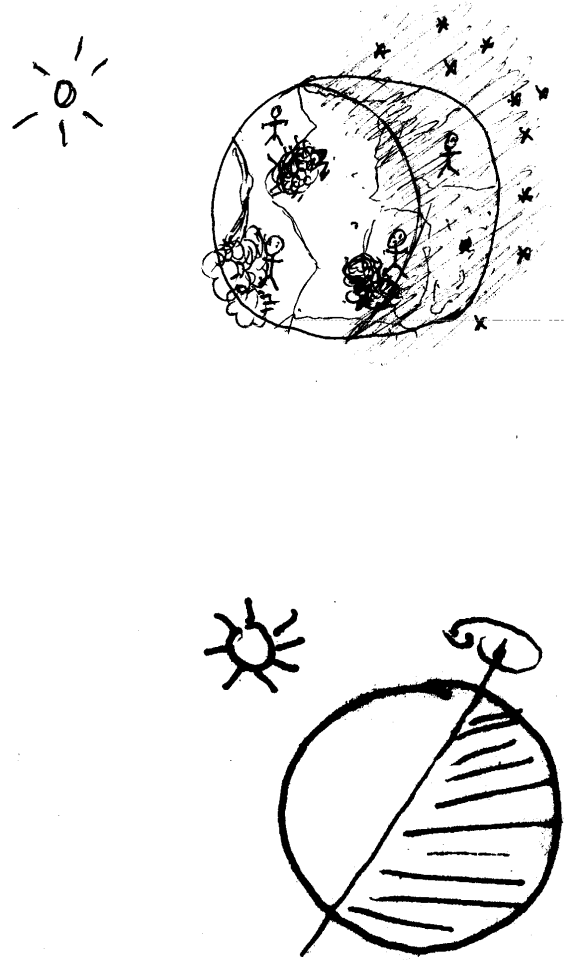

su órbita, como aseguran estos profesores y que, por tanto, es correcto dibujarla, como ellos hacen, aun cuando no tenga nada que ver con el ciclo día-noche. Pero es posible también que la Luna aparezca en los dibujos porque en sus mentes existe una evocadora asociación entre la noche y la Luna, como la del profesor 47, aunque lo cierto es que ello no perturba la explicación correcta $\mathrm{y}$ científica que dan.

El que las estrellas aparezcan de manera irreal e imposible, como las dibuja el profesor 47 (y así lo hacen una veintena más) puede deberse a que el dibujo se realiza desde una doble perspectiva: la Tierra y los continentes aparecen vistos desde el exterior, pero la Luna, el Sol, las estrellas y las nubes se dibujaron vistos desde la Tierra. Es necesario fijarse también en las líneas de puntos que representan el Sol y la Luna en posiciones contrarias a las que tienen cuando se dibujan con trazo continuo: el profesor explica que el día y la noche derivan de la rotación de la Tierra, pero el dibujo revela una reminiscencia de rotación del Sol y la Luna.

\section{Variante c (5 de los 53)}

Este pequeño grupo de profesores da una explicación correcta de cómo se produce el día, pero esa explicación
Figuras 5 y 6

Modelo científico, variante $b$, de los profesores 15 y 47 respectivamente.
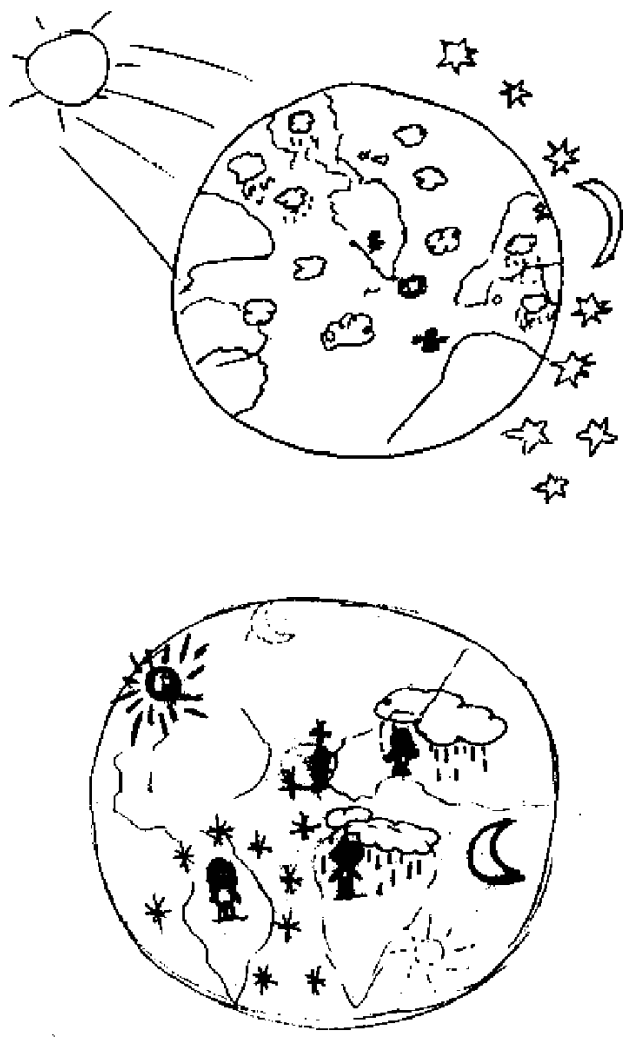

se ve perturbada por una persistente asociación entre la noche y la Luna. En sus dibujos aparece la Luna enfrentada diametralmente al Sol, aseguran que la verán sin duda alguna si miran al cielo por la noche, y que de día estará preferentemente en la otra parte de la Tierra. Se trata probablemente de un modelo de transición entre el científico y el que llamamos de rotación. Para saber cuál de los dos predomina, habría que preguntarles cómo se produce la noche, lo que nosotros no hicimos.

En cualquier caso, todos mantienen, como decimos, una teoría correcta del ciclo día-noche en base a la ausenciapresencia de la luz solar como consecuencia de la rotación terrestre. Aun cuando la analogía día-Sol, nocheLuna no ha desaparecido por completo de las mentes de muchos de ellos, lo cierto es que se puede decir que no perturba la comprensión que tienen, y la explicación que dan, del fenómeno día-noche.

\section{B. Representación rotacional (28 profesores)}

El día tiene que ver con la presencia del Sol y la noche con la de la Luna; la rotación de la Tierra, a la que casi todos consideran esférica aunque parezca plana, permite que en el cielo salgan alternativamente la Luna y el Sol, 
Figuras 7 y 8

Modelo científico, variante $c$, de los profesores 3 y 67. Explican correctamente el ciclo día-noche, pero en sus dibujos la noche aparece asociada a la Luna, situada diametralmente opuesta al Sol. Aseguran que por la noche, si miran al cielo, la verán, sin ninguna duda, y creen que de día está en el otro lado de la Tierra.
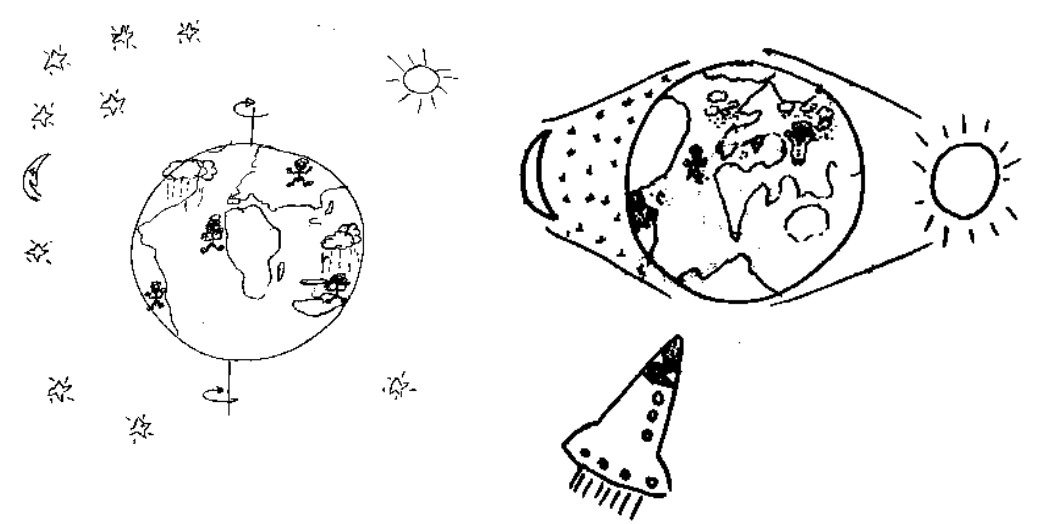

Figuras 9 y 10

Modelo rotacional, de acuerdo con los profesores 6 y 37, respectivamente.
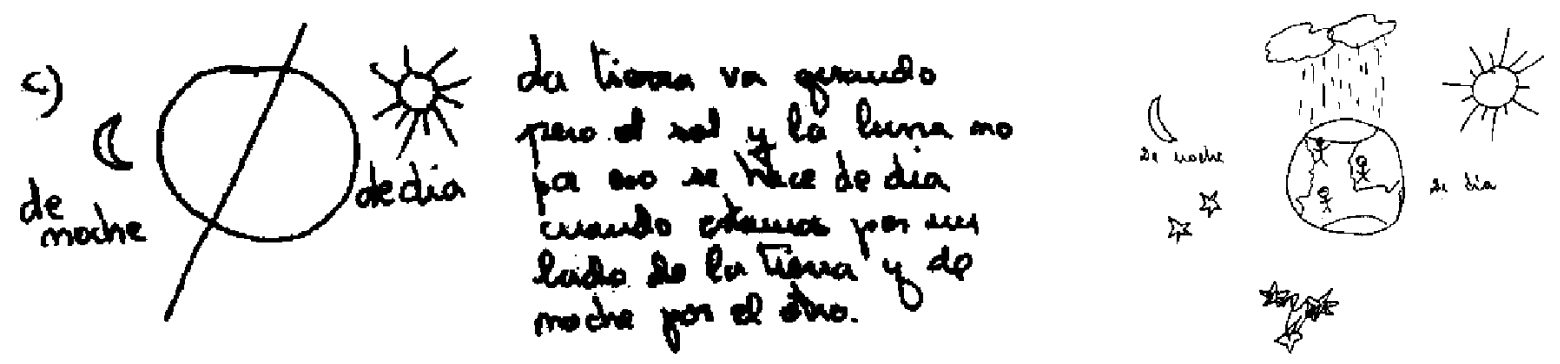

Figura 11

El modelo de revolución explicativo del profesor 65 no necesita comentarios.

Ahora eres un habitante de la tierra y estás en una calle de tu ciudad. Es verano, de noche. Responde a las preguntas.

a) Si miras hacia el cielo qué es lo que verás sin duda alguna. Dime solamente lo que estás seguro que verts. La lura $y$ b estake pobur b) ¿Dónde está el sol? Girando alsededor de la tierra

c) ¿Cómo ocurre eso?

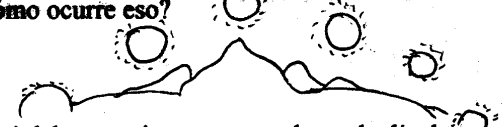

d) ¿Qué debe ocurrir para que se haga de día deñro de unas horas? Que salga \& ofl.

e) ¿Sabes si el sol se mueve? ¿Y la luna? ¿Y la tierra? Sí, claro que permanecen estáticos en uno y otro lado de la Tierra, como expresa el profesor 6. Esta es la principal característica: la Luna y el Sol, diametralmente opuestos, permanecen estáticos, por lo que la rotación de la Tierra los hace aparecer y desaparecer. Días y noches no están relacionados con la presencia-ausencia de la luz solar, sino con la alternativa presencia-ausencia del Sol y de la Luna. Entre estos profesores es mayoritaria la idea de que mirando al cielo por la noche verán sin duda la Luna, pero nunca la verán de día, ya que según ellos está en la otra parte de la Tierra, en donde es de noche.

\section{Representación de revolución (5 profesores)}

El ciclo día-noche se explica por la revolución diaria del Sol y la Luna en torno a la Tierra: el Sol sale por una lado y se pone por el otro, y después le toca el turno a la Luna. Cuando el Sol alumbra es de día; la noche es el tiempo que la luz, más escasa, proviene de la Luna. Para estos profesores, el Sol sale de día; por la noche se va o se oculta. De día, la Luna está en donde es de noche. No aciertan a explicar por qué la Tierra que pisamos parece plana. 
A veces la experiencia diaria y el sentido común chocan con la explicación científica. Algunos profesores, que en su fuero interno mantienen este modelo de revolución, «saben y explican» que día y noche se relacionan con la rotación, pero «saben» también que es el Sol quien se mueve alrededor de la Tierra. Hay 2 profesores que mantienen esa doble explicación.

Figuras 12 y 13

Modelos explicativos de los profesores 48 y 72, respectivamente.
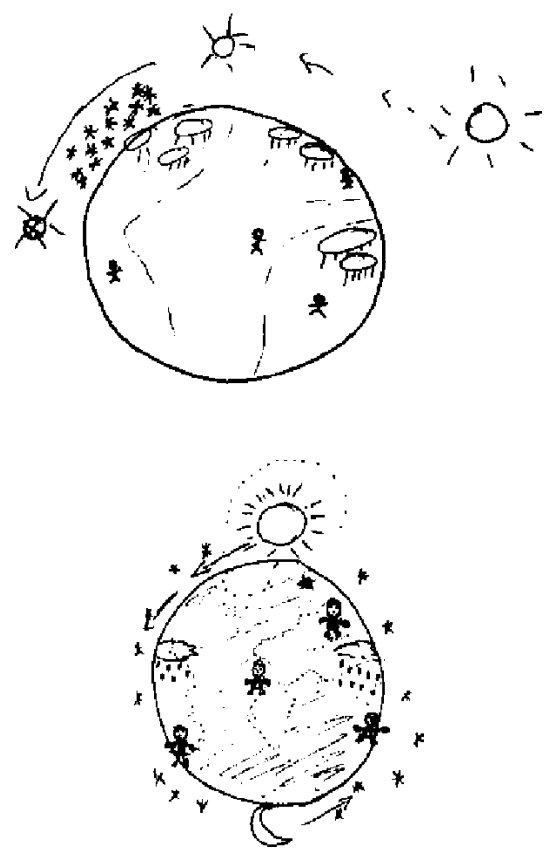

D. Representación de aparición y desaparición del Sol y la Luna (1 profesor)

El día y la noche se derivan de la aparición y desaparición alternativa del Sol y de la Luna, que aparecen y desaparecen detrás de las montañas. Como podemos advertir en el dibujo del profesor 4, la diferencia con el modelo de revolución estriba en que la aparición y desaparición alternativa de los astros no se efectúa mediante una rotación en torno a la Tierra, sino en distintos lugares, en este caso montañas, puesto que el profesor sostiene que la Tierra es plana. Es una concepción muy frecuente en los niños, referida en muchos trabajos, como los de Baxter (1989) y Vosniadou y Brewer (1990, 1994), y que nosotros hemos encontrado también en varios niños de educación infantil de Tenerife, y en algunas madres. Sin embargo, no había aparecido en los estudios realizados con maestros. Bien es verdad que en nuestra muestra solamente se da en este único caso y con una diferencia sustancial en relación con los niños: para éstos la Tierra permanece estática, mientras que el profesor afirma que se mueve.

\section{Figura 14}

Modelo explicativo del profesor 4. No nombra el movimiento de rotación de la Tierra para explicar el día. La Luna está oculta por el día y el Sol por la noche, pero no explica por qué. Para que se haga de día, el Sol tiene que salir e iluminar la Tierra, mientras que la noche se «hace» con la Luna y las estrellas. No obstante, también asegura que ni el Sol ni la Luna se mueven, sino que lo hace la Tierra.

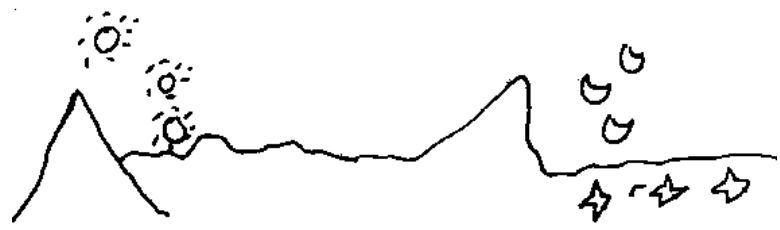

E. Representación de ocultamiento y eclipse (3 profesores)

La noche se produce porque la Luna viene y tapa al Sol, en tanto que por el día la Luna sufre un desplazamiento que permite que el Sol aparezca de nuevo. Se trata de una noción que se manifiesta alguna que otra vez entre los niños, como dan cuenta Sadler (1987), Baxter (1989) y Sharp (1996). Camino (1995) dio también cuenta de esa idea entre maestros y estudiantes de magisterio, aunque se enunciaba de una manera vaga e imprecisa y sin representaciones gráficas. El dibujo y los comentarios del profesor 70 explican perfectamente la idea.

Una variante de este esquema conceptual es el que mantienen los profesores 82 y 94 , pero en este caso el ocultamiento del Sol no se debe sólo a la Luna, sino que es la consecuencia del movimiento combinado de la Tierra y la Luna, como expone el profesor 82, o bien la consecuencia de la traslación terrestre en torno al Sol en un día, que produciría un eclipse de Sol durante toda la noche y un eclipse de Luna que duraría todo el día, como afirma el profesor 94. Ninguno de los dos profesores ha sido capaz de mostrarnos el hecho mediante un dibujo, aunque lo han expresado meridianamente claro con palabras, como las del profesor 82 .

\section{Representaciones y naufragio didáctico}

Las representaciones que llamamos rotacional y de revolución concuerdan con dos de los modelos mentales que Vosniadou y Brewer denominaron sintéticos. En ese sentido, podemos decir que están condicionadas por representaciones sintéticas de la Tierra y por la creencia errónea de que la Luna está implicada causalmente con el ciclo día-noche, creencia que permanece en las mentes de estos profesores desde edades tempranas y que no ha sido resuelta satisfactoriamente a pesar de los estudios de bachillerato y magisterio.

De acuerdo con los modelos mentales propuestos por Vosniadou y Brewer las representaciones de aparición y 
Figura 15

Modelo explicativo del profesor 70. La Tierra y el Sol están estáticos. Por el día la Luna está oculta en el horizonte y deja ver el Sol. Por la noche se desplaza y tapa el Sol. Al amanecer regresa hacia el horizonte (entre las nubes, como manifiesta al preguntarle en dónde se encuentra la Luna de día).

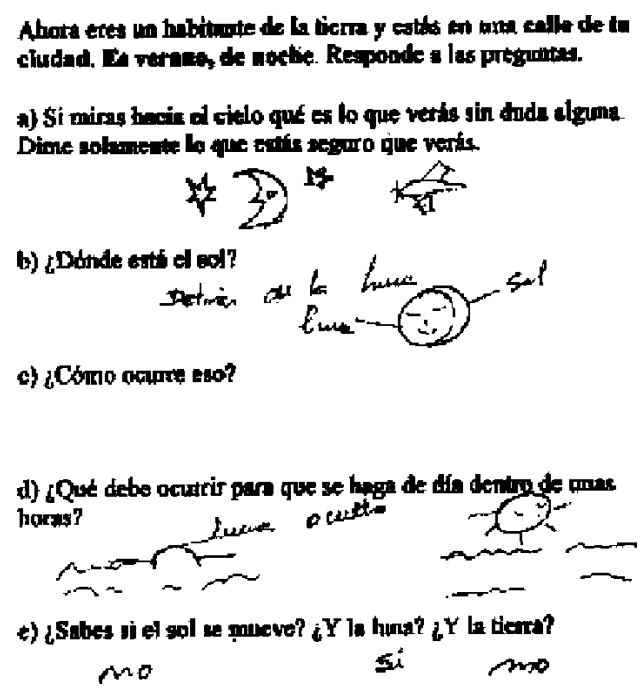

Figura 16 Modelo de ocultamiento, del profesor 82.
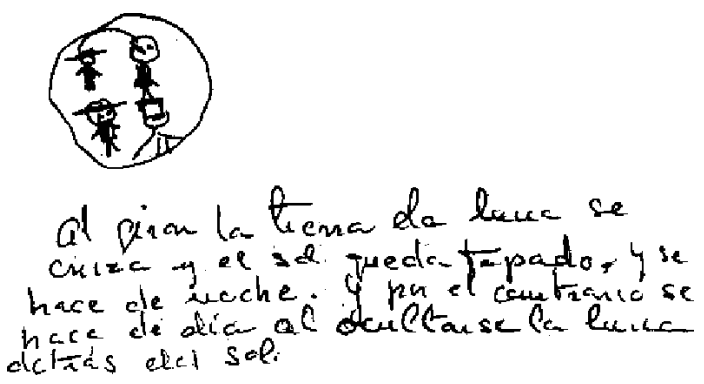

desaparición y de ocultamiento y eclipse se corresponderían con lo que estos autores llaman modelos iniciales, condicionados fundamentalmente por modelos mentales iniciales de representación de la Tierra, determinados, a su vez, por dos presuposiciones: la Tierra es plana e inmóvil; los objetos que no tienen sustento caen. Según Vosniadou y Brewer los modelos mentales iniciales de la Tierra desaparecerían prácticamente al iniciarse la escolarización y al recibir informaciones de que la Tierra es esférica, dejando paso a la formación de modelos mentales sintéticos, como estadios intermedios entre los modelos $\ddagger$ niciales y el científico, con los cuales los estudiantes reconcilian sus creencias y presuposiciones acerca de la Tierra plana y la necesidad de un sostén para los objetos con la nueva información recibida en relación con la forma esférica de la Tierra.

No está claro, por tanto, a qué debemos atribuir la persistencia de las representaciones de aparición y desaparición y de ocultamiento y eclipse en los profesores, puesto que, aun en el caso, poco probable, de que en sus años de escolaridad no hubieran tenido contacto con informaciones acerca de la forma de la Tierra y del ciclo día-noche, sí deben haberlo tenido en el ejercicio de su profesión, pues esos contenidos forman parte del currículo de educación infantil y de primaria. Sí es pertinente señalar que la persistencia de estas últimas representaciones constituyen los restos de un naufragio didáctico, que estos profesores tenderán a reproducir entre sus alumnos y alumnas.

\section{CONCLUSIONES Y RECOMENDACIONES}

«Tenerife tiene seguro de Sol», dice la canción. Y esa circunstancia, añadida a las especiales condiciones del cielo canario, ha favorecido el establecimiento de modernos y sofisticados observatorios astronómicos en nuestro suelo. Por otra parte, aquí tiene su sede uno de los más prestigiosos centros de investigación de astrofísica de nuestro planeta. No deja de ser sorprendente y paradójico que, al parecer de muchos maestros de primaria de Tenerife, también esté asegurada la presencia de la Luna en todas las noches isleñas.

Los resultados de nuestra investigación, a falta de estudios posteriores que confirmen o maticen los datos, son bien desalentadores en la medida en que una tercera parte de los profesores sostienen que la Luna no tiene movimiento y que casi la mitad no son capaces de explicar correctamente algo tan diario -nunca mejor dicho- como el ciclo día-noche. Para los responsables educativos, los datos, más que desalentadores, deben entenderse como espanto que necesita urgente remedio, por cuanto el día y la noche es un contenido básico de la enseñanza primaria, como figura en el desarrollo curricular de la etapa. Un contenido básico que, si no se ha aprehendido, dificultará la posterior comprensión de las fases lunares, las estaciones o la situación de la Tierra en el espacio, por no hablar de la física de la ESO y del bachillerato.

El urgente remedio pasa, indudablemente, por la formación y el reciclaje del profesorado. Pero también por la inaplazable revisión de esos contenidos en nuestros libros de texto, como sostuvimos en otra ocasión (Vega Navarro, 1996). Seguramente tiene razón Lanciano (1989): «[...] lo importante es pensar como Copérnico y mirar al cielo, aunque se vea y se hable como Ptolomeo». Pero siempre que tengamos en cuenta que muchos profesores y diseñadores de libros de texto no hacemos ni lo uno ni lo otro; ni lo contrario: pretendemos hablar como Copérnico, pero como el cielo nos queda tan lejos, en realidad pensamos como aquéllos que condenaron a Atlas a cargar eternamente con la bóveda celeste sobre los hombros. 
Quizás sea hora, por tanto, de aparcar el debate que ha venido consumiendo durante las últimas décadas a la didáctica de las ciencias en torno a las ideas previas del alumnado y de cuál es el mejor modelo de cambio conceptual, para centrarnos en lo que realmente es sustantivo. Porque, ¿de qué habrá de servir este o el otro modelo de cambio conceptual -el de Posner y colaboradores, el de Chi, el de Vosniadou y tantos más- si los materiales curriculares y quienes han de poner en práctica los cambios sostienen las mismas ideas que el alumnado?

\section{AGRADECIMIENTOS}

Como maestra que he sido-que sigo siendo aunque ahora esté en otro lugar-, no pretendo cuestionar la profesionalidad ni la aptitud pedagógica del profesorado que ha dado origen a este trabajo. Al contrario, tengo sobradas razones para afirmar que la mayoría son excelentes profesionales, comprometidos con la mejora de la escuela de Canarias. Quiero agradecer, en ese sentido, la colaboración que me han prestado y la amistad con la que muchos de ellos me honran. Quiero dejar también constancia de la deuda que tengo con Javier Marrero, José Santos, Amador Guarro, Pablo Santana y Esperanza Ceballos, por sus acertadas críticas y sugerencias, que han contribuido a mejorar sustancialmente el original.

\section{REFERENCIAS BIBLIOGRÁFICAS}

AFONSO, R. et al. (1995). Una aproximación a las representaciones del alumnado sobre el universo. Enseñanza de las Ciencias, 13(3), pp. 327-335.

ATWOOD, V.A. y ATWOOD, R.K. (1995). Preservice elementary teacher's conceptions of what causes day and night. School Science and Mathematics, 95, pp. 290-294.

ATWOOD, R.K. y ATWOOD, V.A. (1997).Effects of instruction on preservice elementary teacher's conceptions of the causes of night and day and the seasons. Journal of Science Teacher Education, 8(1), pp. 1-13.

BAXTER, J. (1989). Children's understanding of familiar astronomical events. International Journal of Science Education, 11, pp. 502-513.

CAMINO, N. (1991). Estudio de las ideas de estudiantes de nivel terciario en astronomía. Memorias VII reunión Nacional de Educación en Física. Mendoza. Argentina.

CAMINO, N. (1995). Ideas previas y cambio conceptual en astronomía. Un estudio con maestros de primaria sobre el día y la noche, las estaciones y las fases de la Luna. Enseñanza de las Ciencias, 13(1), pp. 81-96.

COHEN, M. (1982). How can sunlight hit the Moon if we are in the dark?: teacher's concepts of phases of the Moon. Documento presentado en VII Annual Conference on Educational Research. Bloomington. Indiana.

DAI, M.F. y CAPIE, W. (1990). Misconceptions about the Moon held by preservice teachers in Taiwan. Proceedings of the Annual Meeting of the National Association for Research in Science Teaching. Atlanta.

DIAKIDOY, I.A., VOSNIADOU, S. y HAWKS, J.D. (1997). Conceptual change in astronomy: models of the Earth and the day/night cycle in American-Indian children. European Journal of Psychology of Education, 12(2), pp. 159-184.
DICKINSON, V., FLICK, L. y LEDERMAN, N. (1998). Student and teacher conceptions about astronomy: influences on changes in theirideas. Proceedings of the Annual International Conference of the Association for the Education and Teachers in Science. Minneapolis.

FINEGOLD, M. y PUNDAK, D. (1991). A study of change in student's conceptual frameworks in astronomy. Studies in Educational Evaluation, 17(1), pp. 151-166.

FLEER, M. (1997). A cross-cultural study of rural Australian aboriginal children's understandings of night an day. Research in Science Education, 27(1), pp. 101-116.

JONES, B.L.(1988). Primary teacher student's explanations of day and night, the seasons and crescent Moon. Documento presentado en Conference of the New Zealand Association for Research in Education. Nueva Zelanda: Massey University.

JONES, B.L., LYNCH, P. y REESINCK, C. (1987). Children's conceptions of the Earth, Sun and Moon. International Journal of Science Education, 9(1), pp. 43-53.

KLEIN, C.A. (1982). Children's concepts of the Earth and Sun: a cross cultural study. Science Education, 65, pp. 95-107.

LANCIANO, N. (1989). Ver y hablar como Tolomeo y pensar como Copérnico. Enseñanza de las Ciencias, 7(2), pp. 173-182.

MANT, J. y SUMMERS, M. (1993). Some primary-school teacher's understanding of the Earth place in the universe. Research Papers in Education, 8(1), pp. 101-129.

NAVARRETE, A. (1998). Una experiencia de aprendizaje sobre los movimientos del sistema Sol/Tierra/Luna en el contexto de la formación de maestros. Investigación en la Escuela, 34. 
NUSSBAUM, J. y NOVAK, J.D. (1976). An assessment of children's concepts of the Earth utilizing structural interwiews. Science Education, 60, pp. 535-550.

OSBORNE, J., WADSWORTH, P., BLACK, P. y MEADOWS, J.(1994). Space research report: the Earth in space. Liverpool: University Press.

PARKER, J. y HEYWOOD, D. (1998). The Earth and beyond developing primary teachers's understanding of astronomical events. International Journal of Science Education, 20, pp. 503-520.

PIAGET, J. (1926): La représentation du monde chez l'enfant. Madrid: Morata (1984).

RESINES, J.A. y RESINES, N. (1995): Concepciones de los estudiantes sobre astronomía. Actas XIII Encuentro de Didáctica de las Ciencias Experimentales. Madrid: Universidad de Alcalá de Henares.

SADLER, P.M. (1987). Misconceptions in astronomy, en Novack (ed.). Proceedings of the 2nd. International Seminar on Misconceptions and Educational Strategies in Science and Maths, 3, pp. 422-425. Ithaca: Cornell University Press.

SAMARAPUNGAVAN, A., VOSNIADOU, S., BREWER, W.F. (1996). Mental models of the Earth, Sun and Moon: Indian children's cosmologies. Cognitive Development, 11, pp. 491-521.
SCHOON, K.J. (1992). Students alternative conceptions of Earth and space. Journal of Geological Education, 40, pp. 209-214

SCHOON, K.J. (1995). The origin and extent of alternative conceptions in the Earth and space sciences: a survey of preservice elementary teachers. Journal of Elementary Science Education, 7(2), pp. 27-46

SHARP,J.G.(1996). Children's astronomical beliefs: a preliminary study of year 6 children in south-west England. International Journal of Science Education, 18(6), pp. 685-712.

SNEIDER, C.I. y OHADI, M.M. (1998). Unraveling Student's Misconceptions about the Earth shape and gravity. Science Education, 82(2), pp. 265-284.

VEGA NAVARRO, A. (1996). Ideas precopernicanas en nuestros libros de texto. Revista de Educación, 311, pp. 339-354.

VOSNIADOU, S. y BREWER, W.F. (1990). A cross-cultural investigation of children's conception about the Earth, the Sun and the Moon: Greek and American data. (Technical report 497) Urbana Champaign, University of Illinois.

VOSNIADOU, S. y BREWER, W.F. (1992). Mental models of the Earth: a study of conceptual change in childhood. Cognitive Psichology, 24, pp. 535-585.

VOSNIADOU, S. y BREWER, W.F. (1994). Mental models of the day/night cycle. Cognitive Science, 18, pp. 123-283.

[Artículo recibido en septiembre de 1999 y aceptado en mayo de 2000.] 


\section{ANEXO}

\section{CUESTIONARIO}

\section{TAREA 1}

(Las preguntas ocupaban medio folio y en la otra mitad se hacía el dibujo.)

Supón que estás en una nave espacial desde la que se puede ver toda la Tierra mirando desde una ventanilla. Realiza las tareas en el orden indicado.

a) Dibuja la Tierra tal y como piensas que la verías.

b) Dibuja en ella a 4 personas de países diferentes (por ejemplo, de Canarias, Argentina, Rusia, etc.).

c) En un cierto lugar es de día y en otro de noche. Intenta mostrar cómo se hace de noche en el sitio que es de día y de día en donde es de noche. Si tienes muchas dificultades para dibujar esta situación puedes completarla mediante una explicación escrita.

d) En algunos lugares está lloviendo. Dibuja varias nubes y el agua cayendo.

e) Dibuja ahora el cielo estrellado.

\section{TAREA 2}

(Esta tarea ocupaba medio folio. Después de cada pregunta se dejaba espacio para responderla. Lo que aquí está en cursiva también aparecía en cursiva en el texto entregado.)

Ahora eres un habitante de la Tierra y estás en una calle de tu ciudad. Es verano, de noche. Responde a las preguntas.

a) Si miras hacia el cielo, ¿qué es lo que verás sin duda alguna? Dime solamente lo que estás seguro que verás.

b) ¿Dónde está el Sol?

c) ¿Cómo ocurre eso?

d) ¿Qué debe ocurrir para que se haga de día dentro de unas horas?

e) ¿Sabes si el Sol se mueve? ¿Y la Luna? ¿Y la Tierra?

\section{TAREA 3}

(Las preguntas de esta tarea se hacían a continuación de las anteriores. La otra mitad del folio era para el dibujo. Las respuestas a esta tarea no se analizan en este trabajo.)

a) Nuestros antípodas, es decir los que viven en el lado opuesto de la Tierra, están en Australia, en el hemisferio sur. ¿Será allí una noche de verano también? Explícalo.

b) Mediante un dibujo y las aclaraciones escritas que creas conveniente hacer, explica cómo se suceden las estaciones.

\section{TAREA 4}

(Las respuestas a las preguntas 2 y 3 no se analizan en este trabajo.)

1) ¿Por qué la Tierra es plana en el dibujo 1 y esférica en el dibujo 2 ?

(Tarea y dibujo tomado de Sneider y Ohadi, 1998)
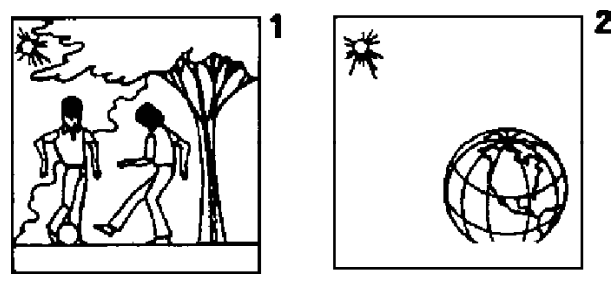
a) Se trata de dos Tierras diferentes.

b) La Tierra es esférica, pero la gente vive en el medio, en una zona plana.

c) La Tierra es esférica, pero tiene partes planas que es donde vivimos.

d) La Tierra es esférica, pero parece plana porque sólo vemos una pequeña parte.

e) La Tierra es plana y redonda: alrededor es redonda pero por encima es plana.

2) En el dibujo se intenta representar la Tierra y tres personas con una piedra en la mano. Las tres personas van a abrir la mano y dejar caer la piedra.

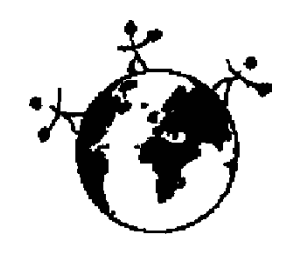

Debes dibujar la trayectoria de la piedra con flechas $(\rightarrow)$.

3) ¿Cuál de los dos modelos utilizarías para construir un globo terráqueo como los que hay en las escuelas, el de la figura 1, el de la figura 2 , o cualquiera es válido?

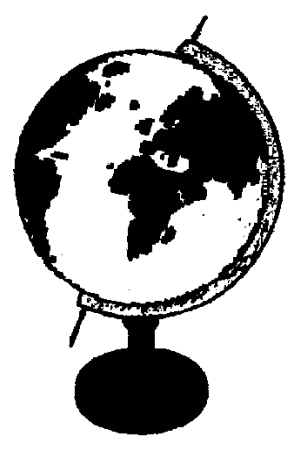

Figura 1

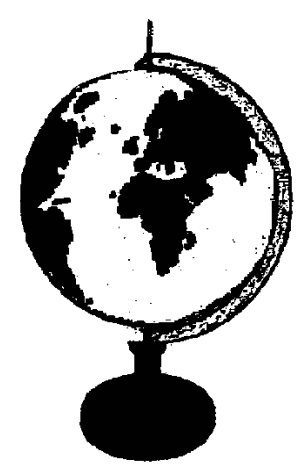

Figura 2

Explica de manera convincente tu respuesta.

4) ¿Dónde está la Luna durante el día? 